\title{
Maladies rares génétiques - Science, soins, éthique
}

\section{Jean Martin}

Dr méd., membre de la rédaction

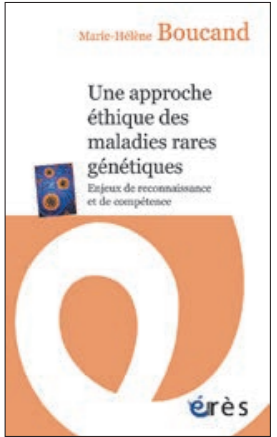

u sujet de cet article voir aussi la contribution de Christine Guckert à la page 1445 de ce numéro.
Références

1 France. Plan national maladies rares 20112014, p. 4 et 34.

Il parle de "guérir de l'idée de guérir" (cf. Martin J. Vivre sans pourquoi. Bulletin des médecins suisses. 2015;96:1042)
Marie-Hélène Boucand

Une approche éthique des maladies rares génétiques

Enjeux de reconnaissance et de compétence

Toulouse: Editions érès; 2018.

357 pages. 19.50 EUR.

ISBN 978-2749258096

Elle-même atteinte d'une maladie rare, Marie-Hélène Boucand a été chef de service aux Hospices civils de Lyon (réadaptation). Son livre traite les multiples dimensions des soins aux personnes atteintes d'une des quelque 7000 maladies rares génétiques connues . Contacts avec le système de soins. "Les retards diagnostiques, la banalisation des symptômes, les jugements formulés suscitent un rapport conflictuel initial avec le corps médical. Période d'errance et de galère» (l'expression d'errance diagnostique a trouvé place dans des rapports officiels français - [1]). «La posture du médecin face aux maladies rares plaide pour une médecine de l'incertitude où il devient possible de dire: 'Je ne sais pas, vos symptômes ne m'évoquent rien de connu, je vais demander un avis spécialisé.'»

L'annonce diagnostique: "Après la dénomination de la maladie, le premier sentiment est souvent celui du soulagement. Le diagnostic permet de comprendre le pourquoi de symptômes qui semblaient venir de nulle part.» Mais ce n'est pas toujours le cas. A plusieurs reprises est évoquée l'épée de Damoclès.

«La transmission est souvent associée à une forte culpabilité de la mère - fantasme de transmission [...] la transmission devient objet de décision alors qu'elle n'est que le fruit du hasard et ne nous appartient pas." «L'enjeu de la reconnaissance est un point fort du vécu; il va, paradoxalement, en émerger une grande force de solidarité [...] Le soin devient alors partagé, tant prodigué par les soignants que par le malade qui, apprenant à prendre soin de lui, peut prendre soin des autres.» «La maladie rare génétique illustre une modernité de la contagion. Elle prend le sens métaphorique de la transmission à un proche [...] Le gène de la science contemporaine vient remplacer le microbe pasteurien.»

Patients-experts, -partenaires, -formateurs. Un chapitre leur est consacré. «Quel chemin parcouru entre la posture paternaliste où le médecin savait ce qui était bon pour 'son' patient sans lui délivrer aucune information, et la conversion marquée par la loi de 2002 [loi Kouchner sur les droits des patients]. Un pas vers un équilibre nouveau.» "Les nouvelles figures de patients, instaurées par les malades du sida et confirmées par ceux touchés par une maladie chronique et/ou rare, c'est la capacité de prendre la parole et de décider.» Les patients-formateurs sont apparus dans la foulée des travaux sur l'éducation thérapeutique de J.-Ph. Assal et A. Lacroix. Noter que, en France, cette compétence fait l'objet depuis 2010 d'une reconnaissance officielle universitaire.

L'auteure à propos des témoins avec qui elle a eu des entretiens approfondis: "Nous avons tâtonné, sans savoir si nous devions les appeler des malades, des porteurs d'un handicap, des usagers, des participants à notre recherche ou des co-chercheurs."

Une médecine de l'adaptabilité. "La médecine ainsi envisagée est celle d'un accompagnement dans le temps pour apprendre comment faire face et résister à l'abattement. Il s'agit d'adaptabilité au sens de Canguilhem, capacité de la personne à retrouver en elle un équilibre au long cours, avec ses nouvelles capacités et limites. Une médecine qui accepte l'échec, lorsque plus rien ne peut être tenté pour guérir.» "A 60 ans, handicapé, j'ai une bonne qualité de vie. Je ne suis plus impatient de guérir" (le philosophe Alexandre Jollien, handicapé lui aussi, a une phrase semblable [2]).

"La désignation des maladies rares semble être celle de l'entre-deux. Il y a dans cette expérience particulière une part de maladie et une part de handicap.» Avec «un point commun: la force paradoxale du manque».

De larges passages sont consacrés à d'autres aspects: le vécu social et familial de ces patients, le «regard des autres", pas toujours aimable; le soutien social en ligne par réseaux et forums; l'utilité des associations de patients (ou de proches). Un chapitre traite de la méthode narrative et de la reconstruction identitaire.

Cet ouvrage est une somme sur les maladies rares génétiques, y compris au plan épistémologique et sociologique. Très bien informé, bien écrit, il apporte une importante contribution, selon la préfacière, «pour avancer sans complaisance dans l'exploration philosophique et humaine d'un monde multiple, mal décrit et mal perçu». 